\title{
Optimization-Based Methods for Revising Train Timetables with Focus on Robustness
}

\author{
FAHIMEH KHOSHNIYAT
}

\section{I1.0 UNMKNONG}


Optimization-Based Methods for Revising Train Timetables with Focus on Robustness

\section{FAHIMEH KHOSHNIYAT}

LIU-TEK-LIC 2016

ISBN 978-91-7685-631-4

ISSN $0280-7971$

Linköping University

Department of Science and Technology

SE-601 74 Norrköping

Printed by LiU Tryck, Linköping, Sweden 2016 


\section{Abstract}

With increase in the use of railway transport, ensuring robustness in railway timetables has never been this important. In a dense railway timetable even a small disturbance can propagate easily and affect trains' arrival and departure times. In a robust timetable small delays are absorbed and knock-on effects are prevented effectively. The aim of this thesis is to study how optimization tools can support the generation of robust railway traffic timetables. We address two Train Timetabling Problems (TTP) and for both problems we apply Mixed Integer Linear Programming (MILP) to solve them from network management perspectives. The first problem is how robustness in a given timetable can be assessed and ensured. To tackle this problem, a headway-based method is introduced. The proposed method is implemented in real timetables and evaluated from performance perspectives. Furthermore, the impact of the proposed method on capacity utilization, heterogeneity and the speed of trains, is monitored. Results show that the proposed method can improve robustness without imposing major changes in timetables. The second problem addressed in the thesis is how robustness can be assessed and maintained in a given timetable when allocating additional traffic and maintenance slots. Different insertion strategies are studied and their consequences on capacity utilization and on the properties of the timetables are analyzed. Two different insertion strategies are considered: i) simultaneous and ii) stepwise insertion. The results show that inserting the additional trains simultaneously usually results in generating more optimal solutions. However, solving this type of problem is computationally challenging. We also observed that the existing robustness metrics cannot capture the essential properties of having more robust timetables. Therefore we proposed measuring Channel Width, Channel Width Forward, Channel Width Behind and Track Switching.

Furthermore, the experimental analysis of the applied MILP model shows that some cases are computationally hard to solve and there is a need to decrease the computation time. Hence several valid inequalities are developed and their effects on the computation time are analyzed.

This thesis contains three papers which are appended. The results of this thesis are of special interests for railway traffic planners and it would support their working process. However, railway traffic operators and passengers also benefit from this study. 



\section{Acknowledgments}

I would like to express my gratitude to my main supervisor, the head of the division for Communications and Transport Systems (KTS), professor Jan Lundgren for his continuous support during my studies at Linköping University. I am sincerely grateful for his providing me this opportunity. I cannot thank enough my supervisor Dr. Johanna Törnquist Krasemann for her brilliant scientific advice and generous support during my research studies. Having the opportunity to work with her is a great honour. Beside my supervisors I would also like to thank my co-author and colleague Dr. Anders Peterson for introducing me to KTS and for his guidance during the writing of the first two included papers.

This study was conducted within the research project "Robust Timetables for Railway Traffic", which is financially supported by grants from Trafikverket (The Swedish Transport Administration), VINNOVA (The Swedish Governmental Agency for Innovation Systems) and SJ AB (a Swedish state-owned operator). I am grateful for all the data provided by Trafikverket. I especially thank our contact person at Trafikverket, Magdalena Grimm.

I have great colleagues at railway/public transport group at KTS, especially my former roommate at the university Tomas Lidén, I am grateful for their valuable comments and sharing their knowledge during our regular group meetings. I also take this opportunity to thank my former colleagues at KTS, Emma Solinen, Gerasimos Loutos, Pavle Kecman, Roya Elyasi and Sara Modarres Razavi for all the scientific and non-scientific discussions we had and for their support during my student life crises.

I am unbelievably lucky to have wonderful and supportive friends around the world, especially in Norrköping, Stockholm, Örebro, Los Angeles, Boston and Tehran, those who showed distance is just a number. Thank you for all the pleasant distractions.

Above all, this thesis is dedicated to my parents, my siblings and to Hamed, Fatemeh, Mohammad, Mobina, Shayan and Sarvin

Norrköping, 2016 Fahimeh Khoshniyat 



\section{Contents}

Abstract

Acknowledgments $\quad \mathrm{V}$

1 Introduction 1

1.1 Motivation 2

1.2 The scope of the research 3

1.3 Problem definition and research questions 3

1.4 Method 5

1.5 Contributions 5

1.6 Publications and presentations 6

$\begin{array}{lll}1.7 & \text { Thesis outline } & 7\end{array}$

2 Optimization Models for Train Timetabling Problem 9

2.1 Existing optimization models for TTP 9

2.1.1 Different types of TTP 10

2.1.2 Planning horizons 11

2.1.3 Level of abstraction 12

2.1.4 Purpose of solving a TTP 15

2.1.5 Mathematical formulations 20

2.2 Remarks on the analyzed previous models 27

2.3 The applied model in this thesis 27

3 Summary of the Included Papers 35

3.1 Paper I 35

3.2 Paper II 37

3.3 Paper III 38 
Contents

4 Conclusions and Future Research 41

4.1 Conclusions 41

4.2 Future research 42

Bibliography $\quad 45$

$\begin{array}{ll}\text { Paper I } & 51\end{array}$

Paper II $\quad 82$

$\begin{array}{ll}\text { Paper III } & 100\end{array}$ 


\section{Chapter 1}

\section{Introduction}

Train timetables are essential components of the railway traffic operations process. They are used for many purposes including dispatcher operations and informing passengers. Train timetables are planned based on the traffic demands (passenger, freight or both), which are requested from railway operators and infrastructure managers. To schedule a train means to calculate the arrival and departure times of the train at stations along its journey, given that the origin-destination and the passing stations along its journey are already known. Track allocations, on lines and at stations, can also be included in the scheduling. The calculated schedule must be conflict-free in the sense that no two trains are allowed to occupy the same infrastructure resource (i.e. track) simultaneously.

The term Train Timetabling Problem (TTP) refers to a problem in which a conflict-free schedule is designed for a given set of trains, running within a given railway infrastructure. There are various types of TTP with respect to planning necessities and requests. In large and dense railway networks, solving a TTP is mathematically challenging. Hence, the need for using computer aided tools is evident. This thesis addresses two different timetabling problems and the application of mathematical tools to solve these problems to optimal or near optimal solutions. The two timetabling problems are as follows:

1) The problem of assessing and ensuring robustness in timetables. The term robustness refers to a property of a timetable where small delays during operations can be absorbed in order to limit the propagation of delays to other trains. In order to ensure 
robustness in a timetable, we first need to assess robustness by measuring relevant robustness metrics.

2) The problem of allocating additional time slots for trains as well as for maintenance in a given master timetable from process and capacity utilization points of view, such that the insertion process has limited impact on capacity utilization. Capacity utilization is referred to as the number of trains running within a selected time period e.g. one hour. A master timetable is designed and finalized typically one year in advance but it needs to be revised when there are new requests for slot allocation. We refer to this problem as on-demand timetabling. The term allocation of a slot refers to assigning an infrastructure resource (i.e. track) for the purpose of operating a train or maintenance work and calculating the start and the end time of its occupation.

\subsection{Motivation}

The use of railway transport has been growing in most West-European countries during the last decade (Eurostat, 2014). The increase in the use of railway transport leads to more dense timetables, in which small disturbances can easily propagate and affect other traffic. To avoid the propagation of delays it is important to ensure that railway timetables are sufficiently robust. This motivates the study of implementing and tuning robustness properties in timetables and evaluating the effectiveness of the proposed robustness metrics. Incorporating robustness properties during timetabling is mathematically challenging.

Furthermore, usually freight train operators cannot fully predict the actual need for access to train slots far in advance. This uncertainty in the prediction usually results in receiving late requests for allocating additional train slots, after an annual master timetable has been finalized. This problem is especially challenging in congested railway networks where passenger trains share tracks with freight trains. Hence, there is a need for a flexible and effective timetabling revision process to handle additional slot requests. This process should be able to allocate available capacity in an efficient manner and handle new requests. The need for revising timetables can occur months in advance, or shortly before operation. The problem of handling late requests can be a significant challenge and needs the use of computeraided tools. 
Both problems mentioned above are computationally demanding and the benefits of using computational scheduling support is evident. The computational decision-support requires in some situations relatively short response time. Hence there is a need to investigate whether the existing optimization models can handle such problems within a short time. When solving the two mentioned timetabling problems, there are cases where applying optimization tools can be too time-consuming. There is therefore a need to investigate and develop boosting methods for speeding up the solving process and decreasing the computation time.

\subsection{The scope of the research}

We tackle the two train timetabling problems from network management perspectives. In both problems addressed, it is assumed that a master timetable already exists. In the first problem, the master timetable needs to be modified in order to satisfy selected robustness properties, and in the second problem the master timetable needs to be modified to meet new requests for additional slot allocations while maintaining the required robustness level, defined by the selected robustness metrics. Another main assumption is that we consider TTP during short term planning and prior to the actual time of the operations. All the case studies in this thesis are based on real timetables which are heterogeneous and non-cyclic.

Rescheduling during the operations, fleet and crew management and long-term planning for maintenance are out of the scope of the thesis.

The results of this thesis are of special interests for railway traffic planners since it can support their working process. However, railway traffic operators and passengers also benefit from the results of this study.

\subsection{Problem definition and research ques- tions}

The aim of this thesis is to study how optimization tools can support the generation of robust railway traffic timetables. Three research 
questions are raised and studied to fulfill this aim.

RQ1: How can we assess and improve robustness in an existing timetable?

To improve robustness in a timetable we first need to assess the robustness. We need to identify quantifiable timetable properties which affect robustness and then identify relevant computational methods which can incorporate these properties in the timetabling process. The literature survey presented in Chapter 2 was conducted to identify the above mentioned properties and methods.

One intuitive method for having a more robust timetable is to increase buffer times and runtime margins. Since increasing buffer and runtime margins will usually lead to higher capacity utilization, it is important to decide where and to which extent extra buffer and runtime margins are needed and should be inserted. We tackle this question with focus on introducing additional headways and observing their implications.

RQ2: How can we assess and maintain robustness in an existing timetable when revising the timetable for allocating additional traffic or maintenance slots?

When inserting additional trains, we first need to investigate whether different insertion strategies result in different revised timetables. Then we need to study various properties of the revised timetables including computation time, robustness and capacity utilization. We also need to investigate whether the existing robustness metrics are beneficial to be used when inserting additional traffic, and how the relevant robustness metrics can be incorporated during the revision process.

We study this question with focus on how optimization methods can be of support.

\section{RQ3: What are the strengths and limitations of the applied optimization model?}

On-demand timetabling is usually requested close to the time of the actual operations. The question is whether the existing optimization models are effective and quick enough for solving on-demand timetabling problems and how we can speed up the solving process 
and decrease the computation time.

\subsection{Method}

The research presented in this thesis relies on optimization-based approaches. An existing Mixed Integer Linear Program (MILP) framework, which is also referred to as Basic Model is applied and developed further. The model is formulated in AMPL and JAVA and the commercial solvers Cplex and Gurobi are applied to solve the corresponding formulated optimization problems.

\subsection{Contributions}

The contributions of this thesis are:

1) A demonstration of a headway-based method for improving railway timetable robustness and also the analysis of the strengths and weaknesses of this method (RQ1).

2) Experimental analysis of the effects of implementing extra headways on heterogeneity, speed and capacity consumption for real timetable instances, showing that an easy and straightforward strategy (TTDSMH) can improve robustness without imposing major changes on the planned timetables (RQ1).

3) Experimental analysis and evaluation of various planning strategies when allocating additional time slots on real life cases, showing that current robustness properties cannot capture the essential needs of a robust timetable when inserting additional traffic and proposing the measurement of Channel Width, Channel Width Forward, Channel Width Behind and Track Switching (RQ2).

4) The identification of the fact that the insertion order, the capacity utilization and the flexibility of the timetabling problem, including flexibility in track allocation and in the insertion time window, influence the feasibility and optimality of the formulated optimization problems when allocating additional slots (RQ2). 
5) An analysis of the computational strengths and weaknesses of a proposed MILP approach based on real life cases, including the development and the assessment of the impact of certain valid inequalities for decreasing the computation time (RQ3).

\subsection{Publications and presentations}

This thesis includes the following papers.

I) Khoshniyat, F., Peterson, A. (2015). Improving Train Service Reliability by Applying an Effective Timetable Robustness Strategy. Submitted to and under the second revision for Journal of Intelligent Transportation Systems, Special issue on Travel Time Reliability.

II) Khoshniyat, F., Peterson, A. (2015). Robustness Improvements in a Train Timetable with Travel Time Dependent Minimum Headways. In proceedings of 6th International Conference on Railway Operations Modelling and Analysis-RailTokyo.

III) Khoshniyat, F., Törnquist Krasemann, J. (2016) An Optimization Approach for On-Demand Railway Slot Allocation. Ongoing research to be submitted for journal publications.

The results of the thesis are also presented in the following conferences and seminars.

- 26th European Conference on Operational Research, EuroInforms Joint International Conference, Rome (2013).

- The joint conferences SOAK (Svenska OperationsanalysKonferensen in Swedish) and NOS6 (The 6th Nordic Optimization Symposium), Gothenburg (2013).

- Transportforum, Linköping (2015).

- 6th International Conference on Railway Operations Modelling and Analysis-RailTokyo, Tokyo (2015).

- Nationell Konferens i Transportforskning, Gothenburg (2013), Norrköping (2014) and Lund (2016). 


\subsection{Thesis outline}

The remainder of this thesis is organized as follows. Chapter 2 presents the most recent and relevant models for various types of TTP and gives a general description of the MILP model applied in this thesis. Chapter 3 summarizes the content of the three included papers. Chapter 4 concludes the main findings of the research presented in this thesis and identifies directions for future research. 



\section{Chapter 2}

\section{Optimization Models for Train Timetabling Problem}

The Train Timetabling Problem (TTP) is a well known problem to the community of railway planners and has been tackled from different perspectives for decades. In a broad context, TTP is defined as finding an optimal feasible timetable for a set of trains while satisfying a set of desired (e.g. operational, capacity, etc.) constraints with respect to some objectives. In this chapter a summary of TTP studies including optimization models for the two timetabling problems addressed in this thesis, is presented and discussed. The terminology used in this thesis is mainly based on those in Kroon et al. (2008b).

\subsection{Existing optimization models for TTP}

The Train Timetabling Problem (TTP), was first formulated as an optimization problem during 60s (Assad, 1980). Since then it has been developed further by many and for various purposes. Models can be categorized differently with respect to different perspectives. Assad (1980) conducted a survey on railway models developed before 1980. He addressed a variety of railway models: freight and passenger modelling, simulation versus optimization, and those developed for lines and networks. Harrod (2012) classifies railway models from math- 
ematical formulation, from cyclic/non-cyclic and also whether track allocation is modelled explicitly. Cacchiani et al. (2014) also categorize models from a mathematical formulation point of view with a focus on real-time rescheduling. Lusby et al. (2009) distinguish between models used for networks vs. lines, whether they cover junction details and/or are cyclic. They summarize various mathematical formulations and solution methods including heuristics approaches for railway track allocation models (Lusby et al., 2009).

In this thesis, models are classified from different aspects including type of TTP, planning horizons, level of abstraction, purpose and mathematical formulations. The most relevant and recent models with focus on i) planning, especially short term planning for non-cyclic heterogeneous timetables, ii) scheduling train time slots, iii) incorporating robustness and iv) allocating additional time slots, are presented in Tables 2.1-2.3. In the mentioned tables the models are ordered according to their position in the hierarchy of planning levels. The rest of the related references are cited in the text wherever applicable.

\subsubsection{Different types of TTP}

Train timetabling problems can be categorized with respect to, i) cyclic (or periodic) vs. non-cyclic (or aperiodic), ii) homogeneous vs. heterogeneous and iii) passenger traffic or freight traffic or mixed. In homogeneous timetables trains have the same profile, i.e. the same speed, running time and stop patterns (Vromans, 2005). Nonhomogeneous timetables are called heterogeneous. In cyclic timetables, all train services are operated with some fixed interval time, the cycle time e.g. one hour, then the schedule is repeated for the total scheduling time window e.g. one day (Vromans, 2005; Erol, 2009). In homogeneous timetables, train profiles (e.g. speed and stop patterns) are similar. Most of the timetables that are designed for passenger traffic are cyclic and homogeneous (e.g. timetables for subway systems). Interested readers are encouraged to read Kroon et al. (2007), Caprara et al. (2007), Kroon et al. (2008a), Liebchen et al. (2010), Heydar et al. (2013). We also refer to Robenek et al. (2014) for a literature review on cyclic and non-cyclic models. However, Robenek et al. (2014) consider TTP from a passengers perspective while we consider it from network management perspectives.

In this thesis we focus on timetabling of mixed (passenger and freight) railway traffic services resulting in heterogeneous and non- 
cyclic timetables. See also Table 2.1.

\subsubsection{Planning horizons}

The planning horizons can be divided into strategic, tactical, operational and real-time rescheduling stages. The most recent and relevant literature with respect to planning horizons can be found in Table 2.1.

Strategic: At the strategic level decisions regarding resource allocation of the infrastructures are made, (i.e. if a line should have a single or double tracks). From TTP perspectives, at this stage train slots are decided and the origin and destination of trains in each corridor are estimated preliminary. The type of the trains (passenger, fast, freight) is also given. However, the routing and the exact departure and arrival times might not have been decided. The auction based models are in this category. In addition to those studies cited in Table 2.1 we can also refer to the work by Klabes (2010) and Perennes (2014).

Tactical: At a tactical level, global routing of trains is planned, but the arrival and departure times are planned only approximately. Deciding the global routing of a train here means to determine the route of a train including origin-destination and important stations but excluding track allocations. At this stage, train slots can be replanned as well, some trains can be cancelled or extra trains can be inserted. Tactical planning is usually made one year in advance.

Operational: On an operational level, the arrival and departure times are planned precisely by considering the operational details, i.e. minimum headways and track allocations at lines and stations. At this level only local routing is allowed. By local routing we mean origin-destinations and important stations for a journey are already decided and are supposed to be fixed but track allocations at lines and at stations are to be planned. Planning at this level can continue until a few days or few hours before the real operations. Usually at this planning level there exists a master timetable that needs to be rescheduled, e.g. when inserting new trains, cancelling trains, allocating time slots for maintenance, etc. Rescheduling at this stage is called offline rescheduling. In addition to those studies cited in Table 2.1 we can also refer to the work by Liebchen et al. (2010) and Caprara et al. (2014).

Rescheduling: Rescheduling can also be done on real-time (during the actual operations). At the real-time rescheduling level the model 
Table 2.1: Classifying models from planning horizon perspectives and the type of TTP

\begin{tabular}{llcc}
\hline No. & Paper & Planning horizon & Type \\
\hline 1 & Caprara et al. (2002) & Strategic & HT-C \\
2 & Borndörfer et al. (2006) & Strategic & HT-NC \\
3 & Borndörfer and Schlechte (2007) & Strategic & HT-NC \\
4 & Borndörfer et al. (2010) & Strategic & HT-NC \\
5 & Harrod (2013) & Strategic & HT-NC \\
6 & Kraay and Harker (1995) & Tactical & F-NC \\
7 & Brännlund et al. (1998) & Tactical & HT-NC \\
8 & Cacchiani et al. (2010) & Tactical & HT-NC \\
9 & Cacchiani et al. (2016) & Tactical & HT-NC \\
10 & Burdett and Kozan (2009) & Tactical & HT-NC \\
11 & Aronsson et al. (2009) & Tactical & HT-NC \\
12 & Forsgren et al. (2013a) & Tactical & HT-NC \\
13 & Gestrelius et al. (2012) & Tactical & HT-NC \\
14 & Forsgren et al. (2013b) & Tactical-operational & HT-NC \\
15 & Meng and Zhou (2014) & Tactical-operational & HT-NC \\
16 & Khoshniyat and Peterson (2015) & Tactical-operational & HT-NC \\
17 & Andersson et al. (2015) & Rescheduling & HT-NC \\
18 & Törnquist and Persson (2007) & Rescheduling & HT-NC \\
19 & Törnquist Krasemann (2012) & Rescheduling & HT-NC \\
20 & Törnquist Krasemann (2015) & Rescheduling & HT-NC \\
21 & Pellegrini et al. (2014) & Rescheduling & HT-NC \\
\hline 2 & Pellegrini et al. (2015) & & \\
\hline
\end{tabular}

HT: heterogeneous, C: Cyclic, NC: non-cyclic, F: freight line

is supposed to handle disturbances effectively and quickly. Models that are developed for real-time rescheduling can usually handle offline rescheduling as well but they have different objectives. Some of the studies tackling real-time rescheduling problems in addition to those studies cited in Table 2.1 are Lamorgese and Mannino (2013), Lamorgese and Mannino (2015), Cacchiani et al. (2014), Louwerse and Huisman (2014) and Acuña-Agost (2010).

From the above definitions it is clear that there are some overlaps between different planning stages. Some details that are necessary for operational purposes can also be included at the tactical planning stage. Maintenance work and train service connections can be included in all the planning stages with different levels of detail. However, maintenance work, train service connections, the feasibility of the train movements at stations, as well as crew and fleet scheduling are beyond the scope of this thesis. For further information regarding planning for maintenance an interested reader is encouraged to read Budai-Balke (2009) and Lidén (2016).

\subsubsection{Level of abstraction}

Enlarging the size of TTP by including more planning details, i.e. more variables, may lead to a computationally hard problem. To han- 
dle the complexity issues, TTP models can be developed such that at each planning level only essential planning details are covered. Models can be abstracted from different perspectives which are described in the following paragraphs and also marked in Table 2.2. Note that the model specifications in Table 2.2 are not only based on model formulations but also based on the experimental analysis of the case studies. If a model is potentially capable of handling networks as well as lines but it is tested only for line cases then it is marked under column line. Accordingly, if a model is potentially capable of handling bidirectional traffic but is tested only for unidirectional cases then it is marked as unidirectional.

\section{Network vs. corridor (line)}

Some models can be applied to railway networks while some can be used for corridors. Lines are those components of a railway network that connect two stations. A line can have a single or multiple tracks. Usually in the early stages of planning, networks are considered while detailed planning is often limited to corridors. Considering operational details in large networks is computationally demanding. Limiting the problem geographically, may provide the opportunity to include more operational details.

\section{Global vs. local routing}

Here we repeat the definitions for global and local routing from section 2.1.2. Deciding the global routing of a train means to determine the route of a train including origin-destination and important stations but excluding track allocations. By local routing we mean origindestinations and important stations for a journey are already decided and are supposed to be fixed but track allocations at lines and at stations are to be planned. When there is a master timetable, the terms global and local rerouteing are used instead.

\section{Train Slots (TS) vs. Train Time Slots (TTS)}

To plan a Train Slot (TS), also in literature referred to as train itinerary or a train path, is to decide whether a train with a fixed global routing should be operated within a particular time window or not. However, the exact departure and arrival times at stations along its journey might not necessarily be planned. On the other hand, 
planning for a Train Time Slot (TTS) means that all the arrival and departure times at all the stations along its journey should be decided. When considering train time slots (TTS) there is usually a master or ideal timetable with approximate arrival and departure times already planned and the objective is usually to plan the exact arrival and departure times and to generate a conflict-free timetable while deviations from the master timetable is as little as possible. Depending on the planning purposes, one or both of TS and TTS can be included during the timetabling process.

\section{Train precedence}

When the order of running trains is given, e.g. when there is a master timetable, then after replanning or rescheduling, the order of trains might change. This change can be modelled explicitly or implicitly. Modelling the order of trains explicitly increases the number of variables. Nonetheless, it provides the ability to control the reordering of trains. For instance, by limiting the number of preceding trains that a train is allowed to overtake, the size of the TTP can be decreased considerably.

\section{Track allocation on lines vs. at stations}

Some models only consider TTP on lines and they assume that the capacity at stations is satisfactory. Some models consider the capacity at stations at macroscopic levels, i.e. considering the number of trains being present at a station simultaneously. On the other hand, there are models that are mainly developed for allocating tracks at stations, see Zwaneveld et al. (1996), Kroon et al. (1997), Dewilde et al. (2013). There are also models that capture both aspects.

\section{Bidirectional vs. unidirectional tracks}

Tracks are operated either bidirectionally or unidirectionally. In many countries bidirectional tracks are not allowed because of the limitations of signalling systems. Intuitively, the operation of bidirectional multiple tracks influences the size of the corresponding optimization problem mathematically. Since the number of alternative train routes may increase when additional tracks are available. 


\section{Infrastructural detail}

Models can be categorized by the level of the infrastructural details that they can cover. Some models only consider links and nodes representing lines and stations. This type of modelling is suitable for tactical planning level since it does not capture the operational details.

Some models consider the details of signalling systems including blocks as well. If a line section has multiple block sections, it can be simultaneously used by two trains, running in the same direction, provided that they are separated by a minimum headway for that particular section (Andersson, 2014). Access to blocks is controlled by signals.

In addition to the above, some detailed models can capture the details of track circuits as well. Track circuits are the smallest components of the railway infrastructure for detecting the presence of a train on a track. On track circuits the presence of a train is automatically detected. A block section is a sequences of several track circuits (Pellegrini et al., 2015). Capturing this level of detail is useful especially during real-time rescheduling.

\subsubsection{Purpose of solving a TTP}

The general purpose of solving a TTP is to generate a conflict-free timetable. Beside that, there can be other purposes as well. These purposes can be either stated in the objective function or formulated as constraints in a TTP. Some of these purposes are: rescheduling, global/local rerouting, planning for maintenance, as well as ensuring robustness and handling on-demand timetabling. We focus more on the optimization models that incorporate robustness and also those that are used for inserting additional trains.

\section{Assessing and ensuring robustness}

To improve robustness in timetables there are several methods proposed in the literature. The proposed methods are mainly based on:

i) Increasing or re-allocating runtime margins in order to absorb delays. Runtime margins are extra times included in the travel time of trains running between two consecutive stations. 
ii) Increasing or re-allocating buffer times or headways in order to reduce knock-on effects. Buffer time is the extra time to the technical minimum headway between two trains at stations. Headway is the time distance between the departure of two trains. A headway value is an indication of dependencies between two trains.

iii) Increasing flexibility in timetables. Flexibility in a timetable can refer to flexibility in departure and arrival times or flexibility in changing the order of trains.

Headway and buffer times are two of the important components in creating robust timetables. Swedish Transport Administration has published the general rules for minimum headways in different corridors (Konrad, 2014). According to Edbring (2014) in some stretches where the demand is high, minimum headways can be replaced by technical minimum headways (the amount of headway that is technically needed for safe operations). This reduction can have negative effects on the robustness of timetables.

Before improving robustness we first need to asses robustness by using some quantifiable metrics. We distinguish between metrics that are measured in timetables before operations (ex-ante) and those that are measured after the operation (ex-post, also called performance indicators or performance measures). A recent review of ex-ante robustness metrics can be found in Andersson (2014) and in the included papers I and III. A summary of performance measures can be found in paper II. In this thesis we focus on finding a headway-based method for improving robustness in timetables (ex-ante) and then we evaluate the proposed method ex-post.

Some of the most important and relevant existing robustness metrics in the research presented in this thesis are:

- TAoRM: Total Amount of Runtime Margins in a timetable. This metric specifies the sum of all runtime margins at all line sections during the journey for all trains (Salido et al., 2008).

- WAD: Weighted Average Distance calculated for each train. It measures the average distance between the location of the runtime margin and the origin of a train (Vromans, 2005). A value smaller than 0.5 indicates that the majority of runtime margin is allocated in the first half of the journey. 
- PoH: Percentage of Headway equal to the technical minimum headway. Introduced by Andersson et al. (2013) and inspired by Kroon et al. (2008c) this metric specifies the percentage of the headway which is equal to the minimum headway. This can be calculated for each train separately or for all the trains in a timetable.

- SSHR: Sum of Shortest Headway Reciprocals to measure heterogeneity. Introduced by Vromans et al. (2006) this metric specifies the sum of the inverse of the shortest headway between each pair of trains which run in the same direction in each section (per track, per direction). According to Vromans et al. (2006), a timetable is more homogeneous and robust for the smaller values of SSHR.

The most recent and relevant literature on the use of headway and buffer times to improve robustness can be found in paper I. One recent paper on robustness methods, that is not mentioned in the included papers, is the work by Jovanović et al. (2016) where the distribution of buffer times is defined as a general Knapsack problem in which the total buffer time in a timetable is the capacity of the Knapsack. In the same study, the distribution of buffer times is based on the priority of locations with respect to delay sensitivity and delay propagation. One important aspect of the study by Jovanović et al. (2016) is that in order to reduce the complexity of the problem, the optimization model for robustness is separated from optimization model for timetabling.

\section{Inserting additional traffic}

The problem of inserting additional trains in an existing timetable has received rather limited attention so far in the literature. A full summary of the studies related is presented in paper III.

Forsgren et al. (2012) studied a case where a new train is inserted into a timetable and is supposed to be able to compete with the bus service running between the same origin-destination. They illustrated how the flexibility in the existing timetables can influence the final solution. Aronsson (2014) introduced the term incremental allocation (Swedish: Successiv tilldelning) of train slots and how it can affect the capacity utilization i.e. in terms of the number of running trains, during ad-hoc planning for timetables. 
Cacchiani et al. (2010) consider inserting additional freight trains into an existing timetable where the schedule of existing passenger trains is kept unchanged but the existing freight trains can deviate from the master timetable within a defined time interval. The experimental analysis is done for double tracks. Track allocation at stations is respected only by checking the number of trains that are present simultaneously at each station. In Cacchiani et al. (2016) the method is extended by allowing trains to be cancelled based on different cost functions.

Burdett and Kozan (2009) consider various levels of flexibility in the timetables by inserting additional trains in a single track line where cancelling trains is not allowed. They define the level of flexibility as unrestricted, loose and tight based on the size of the time window that the existing trains can deviate from the master timetable. They also consider track allocation at stations only with respect to capacity limitations and the total number of trains at the stations simultaneously. 


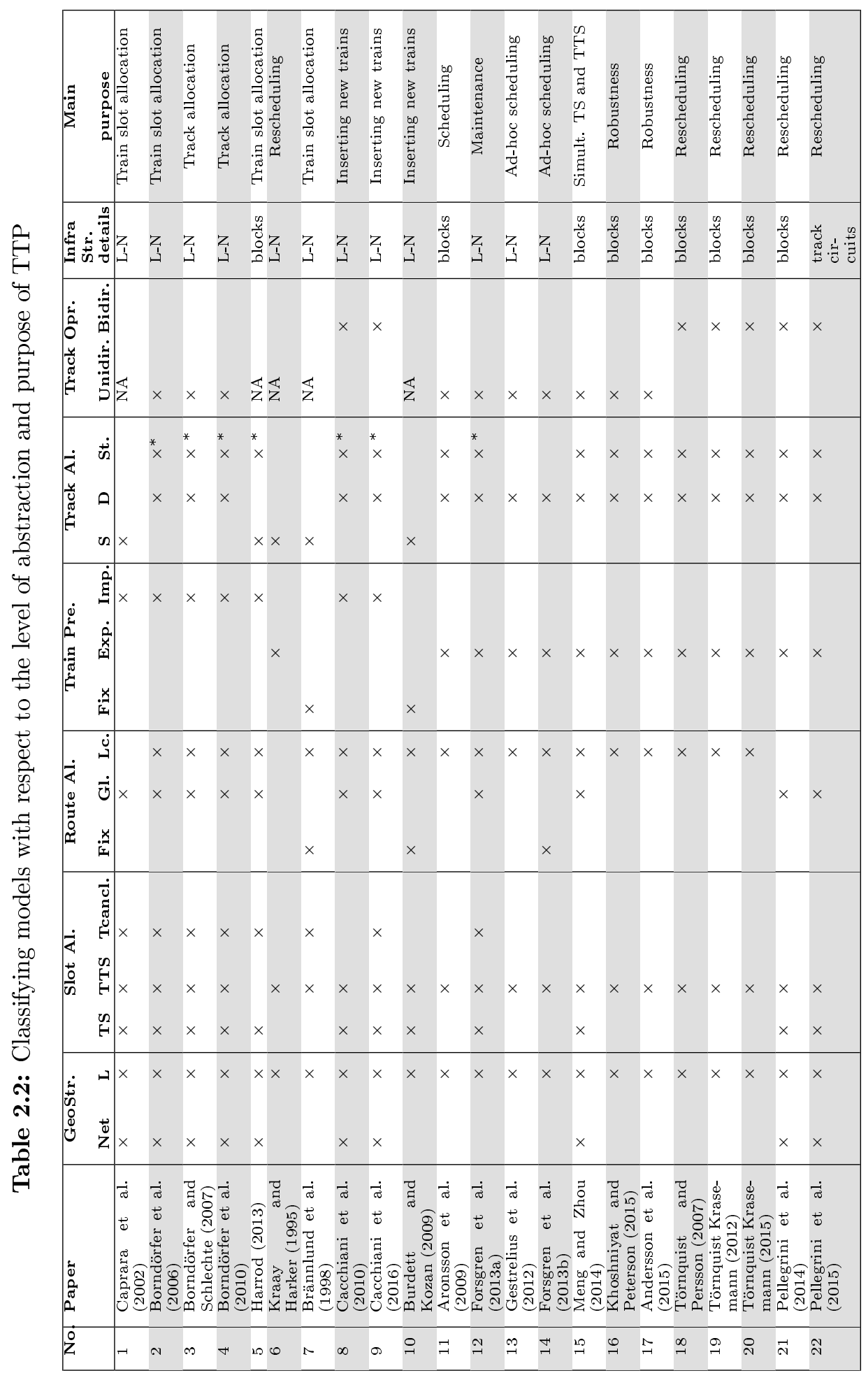

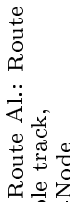

ธี่

$\stackrel{\oplus \ddot{z}}{\Rightarrow}$

品

ర๊

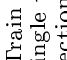

$\because \ddot{\oplus}: \overrightarrow{0}$

藏.

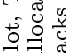

क⿺

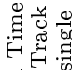

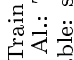

$\ddot{H}$ 我.

난

学苦若

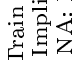

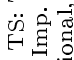

है

\%:

希.

훙.ำ

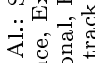

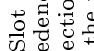

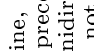

글

हैं

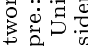

そ.

苟苟

की

鸷

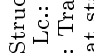

ฮె

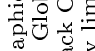

渮

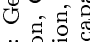

究要

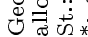




\subsubsection{Mathematical formulations}

Different mathematical formulations can be applied to railway timetabling problems. The most frequently used formulations are Integer Programming (IP), Binary Programming (BP), Linear Programming (LP), Mixed Integer Linear Programming (MILP), graph theory and maxplus. In Table 2.3 the mathematical details of the relevant models including formulation, variables, objective functions, solution methods, case studies and their corresponding computation time, are presented. By looking at Table 2.3 one can observe that there is a relation between the choice of mathematical formulation and the planning horizon. For instance, in order to determine train slots, graph theory and IP (BP) formulations are applied in several studies. While scheduling train time slots, at short term planning and operational levels, the use of MILP models are very common. The reason is that the size of the integer problem increases significantly when covering higher levels of detail especially in the case of large instances. Moreover, MILP models allow the train precedence to be formulated explicitly which is more desirable for short term planning and rescheduling since the explicit formulation provides a better control over the order of trains if needed. In addition to those models mentioned in Table 2.3, the application of a MILP model for connected public transport schedules (for bus and trains) can be found in Schöbel (2001).

In the following paragraphs we refer to the most relevant surveys in TTP formulations.

Lusby et al. (2009) conducted a survey on various track allocation models. They categorized models by line, network and junction train routing. They also categorized models based on common mathematical formulations including conflict graph, node packing, graph colouring, constraint programming and heuristic approaches.

Erol (2009) performed some experiments on different models including and excluding explicit track allocation at the stations. He concluded that for a set of real and artificial data with maximum of 37 stations, including stations in the modelling is too time consuming and after 4 hours the optimal solution was not found using a noncommercial solver or Cplex (remaining gap 2\%).

Mannino (2011) compared two representation of station capacity, non-compact formulation vs. compact formulation, of a MILP model for single track lines. Both formulations could solve real-life instances while the non-compact behaved slightly better in computational time. 
Cacchiani and Toth (2012) describe traditional TTP as nominal TTP and compare it with models on robust TTP. They compare different TTP models from various perspectives including, network vs. corridor, freight vs. passenger, cyclic vs. non-cyclic.

Harrod and Schlechte (2013) compared two different mathematical representations of TTP. One based on aggregated fixed time separation on tracks and the other based on the actual physical occupation at blocks, transformed to times. They applied both formulations on the same set of problems and concluded that while there are significant differences in the computation time for some selected instances, both formulations are comparable in aggregate and in nearly half of the scenarios the solutions were identical.

At the real time rescheduling levels researchers have developed different formulations to tackle timetabling problems in less time. Lagrangian relaxation formulations were more common in the past but nowadays decomposition formulations are becoming more common.

\section{Solution methods}

Exact or approximate methods can be used to solve optimization problems. Commercial packages including Cplex and Gurobi are mainly based on exact methods (branch and bound) and are reported to find solutions sufficiently fast for small and medium sized timetabling problems at the strategic and tactical levels. In large cases using approximate methods including heuristic approaches are more common. Comparing models from computation time should be done with respect to all the assumptions including the flexibilities and restrictions of the models as well as the specifications of the test cases. 
Chapter 2. Optimization Models for Train Timetabling Problem

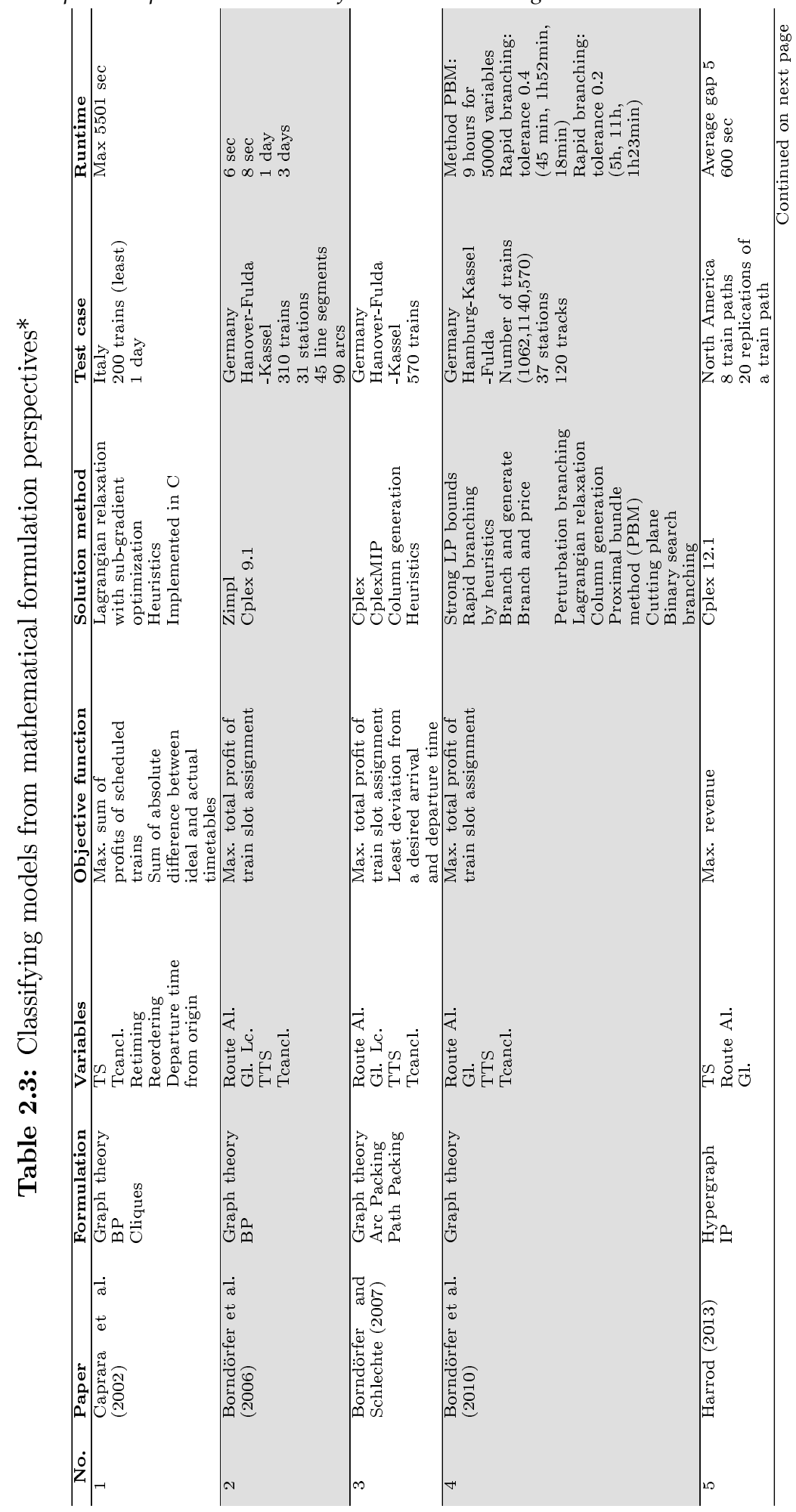


2.1. Existing optimization models for TTP

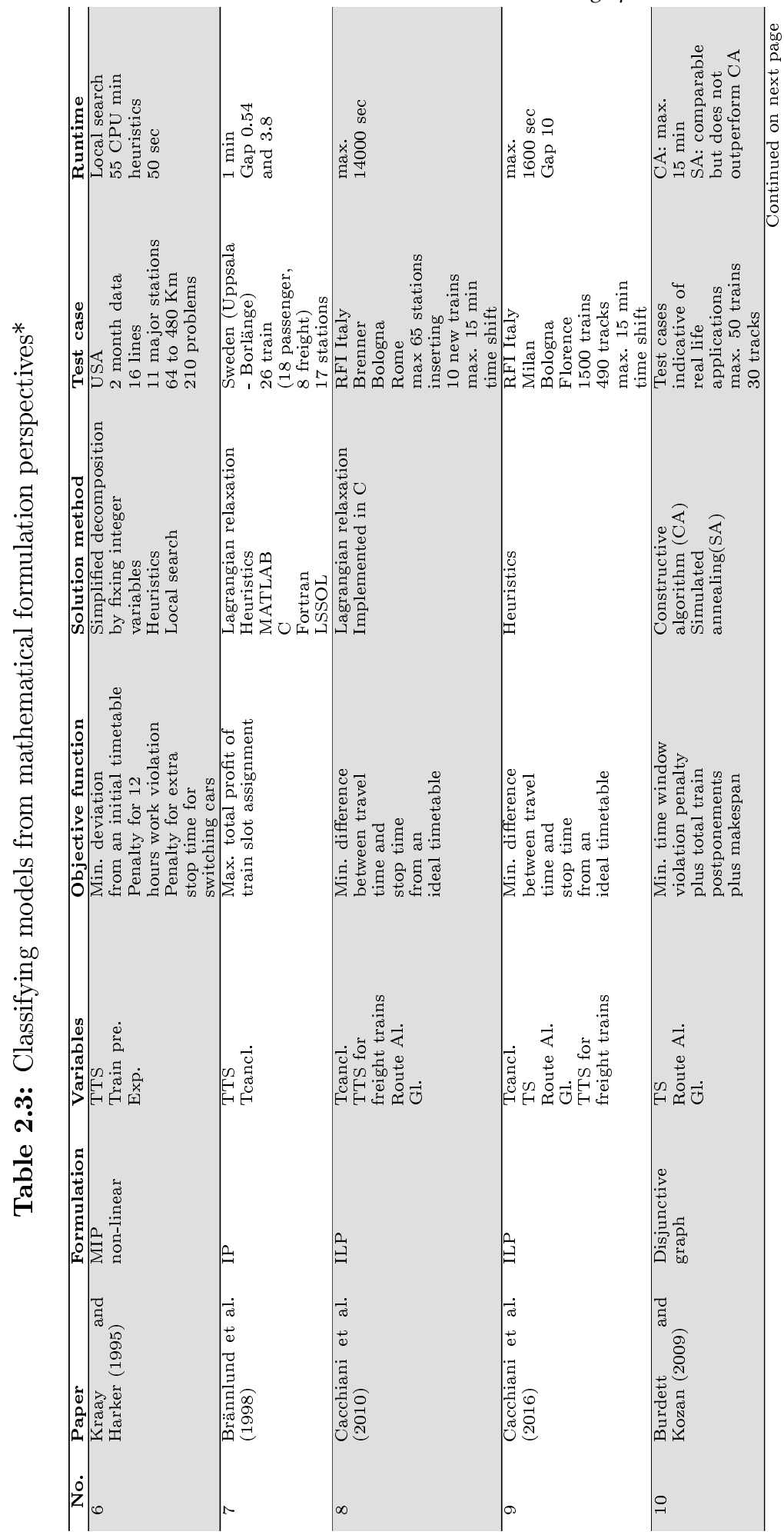


Chapter 2. Optimization Models for Train Timetabling Problem

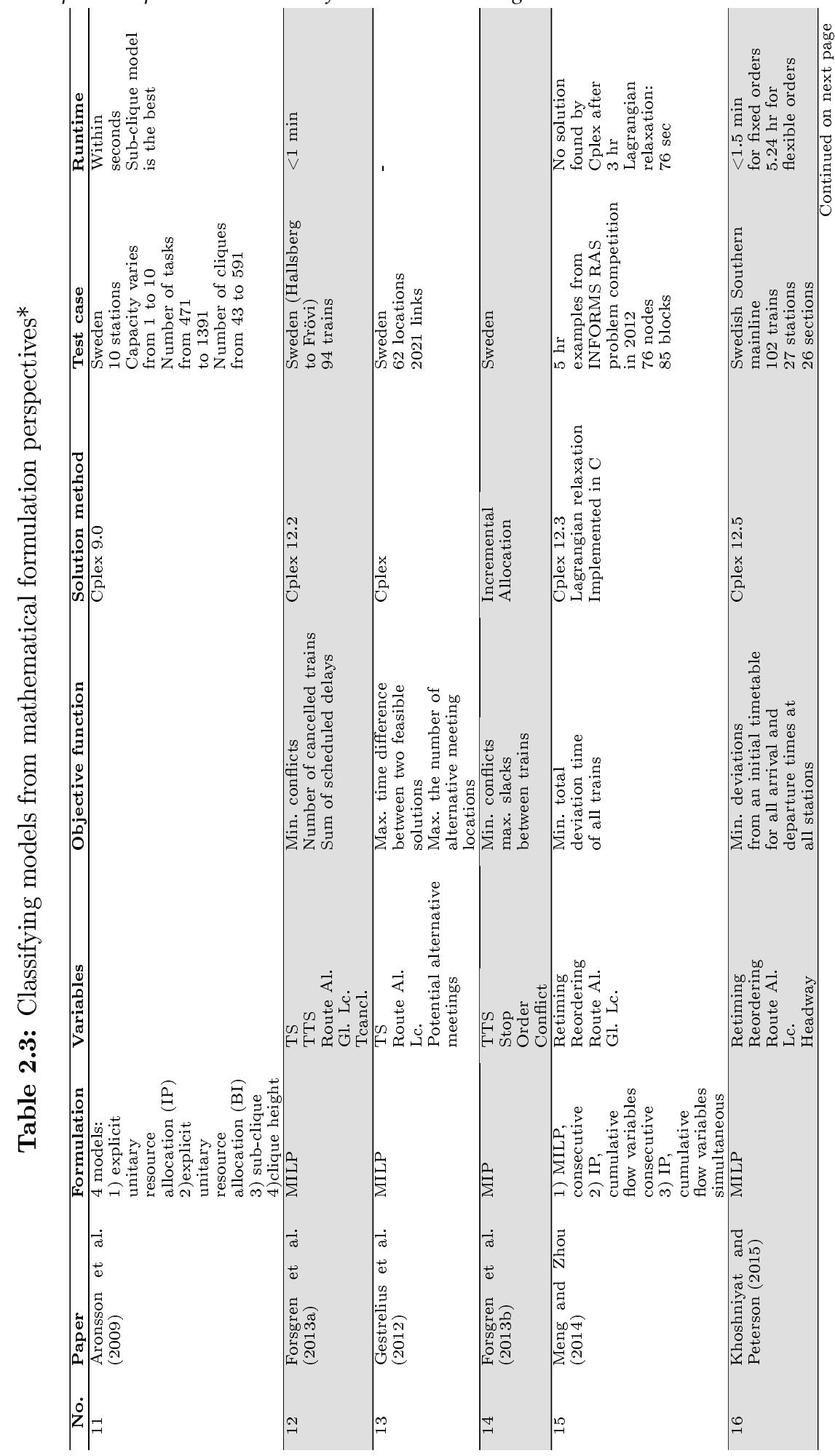




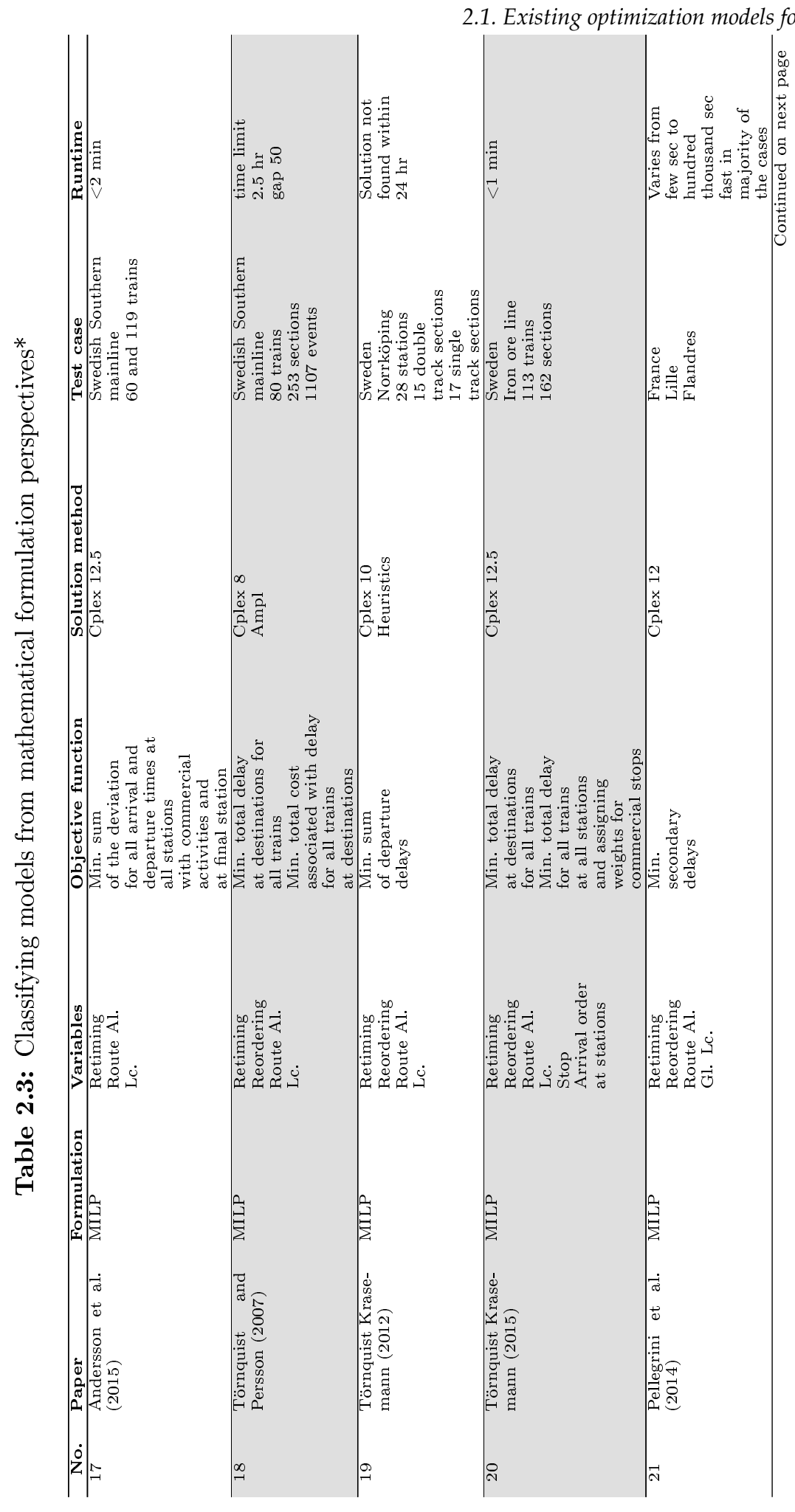


Chapter 2. Optimization Models for Train Timetabling Problem

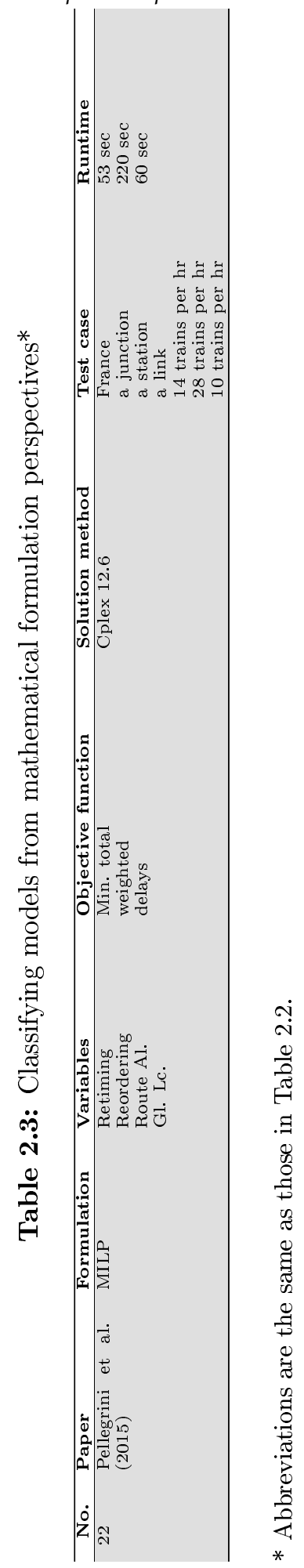




\subsection{Remarks on the analyzed previous mod- els}

The models discussed in the previous section are, to the best of our knowledge, the most relevant TTP models with focus on short term planning, scheduling train time slots, incorporating robustness and allocating additional time slots.

Planning various properties of timetables simultaneously, e.g. planning train slots and train time slots at the same time or planning global and local routing simultaneously, usually leads to have better optimal solutions. Yet in practice it is not always possible, given how timetabling problems are computationally hard. As the time of the operation approaches, planning for more operational details as well as revising the timetables might be requested. Some studies try to include as much detail as possible during the modelling while some try to reduce unnecessary flexibilities in the models to decrease the size of the timetabling problems. Having a balance in-between is the key to have an optimal planning with respect to the computational limits.

For the purpose of this thesis we are interested in a model that was developed for non-cyclic heterogeneous traffic and can be applied for short term and operational planning horizons. The model should include operational detail i.e. local routing and explicit modelling of track allocation at stations as well as track allocation in multi-track lines under bidirectional operations to ensure capacity limitations with respect to the number of available tracks as well as track and platform lengths. It should also consider train precedences explicitly to provide the control over changing the order of trains if necessary.

The model developed by Törnquist and Persson (2007) and its recent version applied in Törnquist Krasemann (2015), is used for the analysis in this thesis since it holds the necessary planning details and it is developed for the Swedish context.

\subsection{The applied model in this thesis}

A general description of the applied model is presented in this section. A complete description of the model can be found in the corresponding appendices in each of the included papers. 
The model is a mixed integer linear program (MILP) formulated as an event-based description of railway traffic. An event represents a train $i$ passing a section $j$ while occupying track $q$. We distinguish between train event $s$ and section event $k$. To denote a train involved in the $k^{\text {th }}$ event of section $j$, we write $i_{(j, k)}$ and we let $s_{(j, k)}$ denote the corresponding train event. Given a train $i$ and its train event $s$, we identify the corresponding section as $j_{(i, s)}$. Basically, train and section events are two different representations of the same event.

\section{Sets and parameters:}

A list of the parameters used in the model is presented here.

$T$ : set of trains

$J$ : set of sections

$S_{i}$ : ordered set of events for train $i \in T$, it contains all the section numbers traversed by train $i$

$K_{j}$ : ordered set of events for section $j \in J$, it contains all the train numbers that pass section $j$

$C_{j}$ : number of tracks at each station $j$

$\hat{t}_{(i, s)}^{B}$ : initial start time of train $i \in T$ at event $s \in S_{i}$

$\hat{t}_{(i, s)}^{E}$ : initial end time of $i \in T$ at event $s \in S_{i}$

$t_{j}^{c}$ : clearance time at section $j$

$t_{(i, s)}^{\text {min }}$ : minimum time required for train $i$ to complete event $s$

$q_{(i, s)}$ : track number assigned to train $i$ at train event $s$

$M$ : sufficiently large constant

$h_{m i n}$ : technical minimum headway time between any two consecutive trains $i$ and $i+1$ on a section

\section{Variables for arrival and departure times, track allocation and train precedences:}

Each train event $s$, related to train $i$, has two continuous variables, one for the start time $t_{(i, s)}^{B}$ and one for the end time $t_{(i, s)}^{E}$ of the event and one binary variable $x_{(i, s, q)}$ which takes value of one if track $q$ is allocated for train event $s$. Each section event $k$, related to section $j$, has two binary variables for the precedence of trains. In single track lines, the overlap between section events is not allowed, hence there are only two situations allowed after rescheduling: i) either the order of two events is the same as the initial order or ii) the order is changed. A binary variable $\gamma_{(j, k, v)}$ takes value one if the order between 
two section events $k$ and $k+v$ is the same as the initial order and zero otherwise. The initial order of trains are based on the master timetable. In one section with multiple tracks, events may overlap if they are allocated different tracks, so there is not only a binary choice but multiple alternative scenarios. This is elaborated further in Figure 2.1 since understanding the concept and relations between these variables is the key to generating some of the valid inequalities analyzed in paper III. 


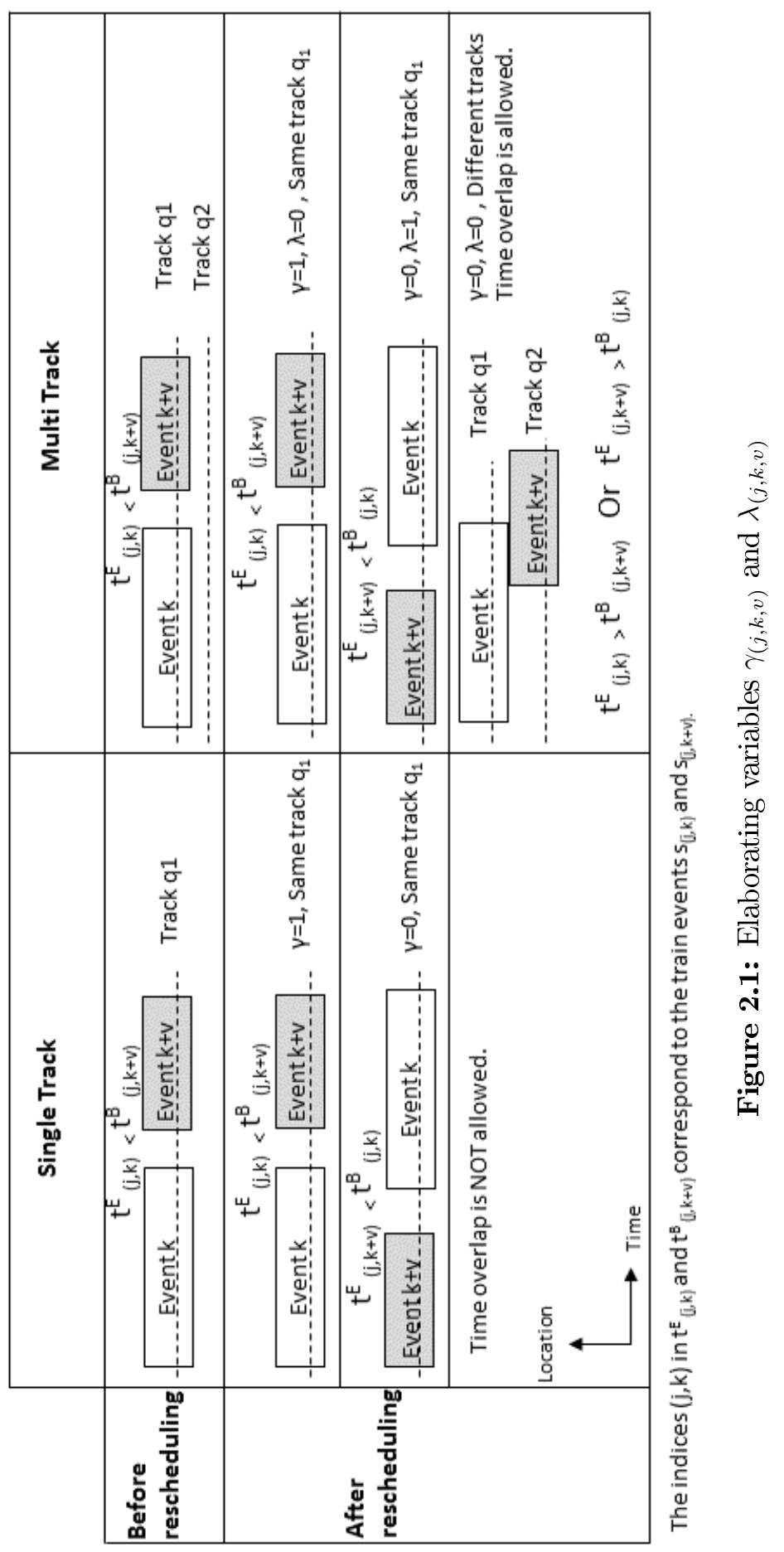


In multi track lines, two section events $k$ and $k+v$ either 1) keep their initial order, or 2) switch the order or 3) have time overlap. Hence, a new binary variable $\lambda_{(j, k, v)}$ is introduced. In multi track lines, $\lambda_{(j, k, v)}$ takes value zero if the order between two section events $k$ and $k+v$ is the same as the initial order and one if the order is swapped. By applying constraint $2.1, \gamma_{(j, k, v)}$ and $\lambda_{(j, k, v)}$ cannot take value one simultaneously. By applying constraint 2.2 we make sure that where on the same track, either $\gamma_{(j, k, v)}$ or $\lambda_{(j, k, v)}$ takes value one. By this formulation $\gamma_{(j, k, v)}$ and $\lambda_{(j, k, v)}$ both can take value zero simultaneously only when they are not happening on the same track. In this situation the events $k$ and $k+v$ can be overlapped. Worth repeating is that each train event is mapped to exactly one section event and vice versa.

$$
\begin{gathered}
\lambda_{(j, k, v)}+\gamma_{(j, k, v)} \leq 1 \quad C_{j} \geq 2 \\
x_{\left(i_{(j, k+v)}, s_{(j, k+v)}, q_{(i, s)}\right)}+x_{\left(i_{(j, k)}, s_{(j, k)}, q_{(i, s)}\right)} \leq \gamma_{(j, k, v)}+\lambda_{(j, k, v)}+1
\end{gathered}
$$

\section{Objective function:}

The objective function can be adjusted for different purposes. In this study the objective function aims at minimizing the sum of deviations between the initial timetable and the new calculated timetable at departures and arrivals for all the trains in all the sections along their journey.

$$
\min \sum_{i \in T} \sum_{s \in S_{i}}\left(d_{(i, s)}+a_{(i, s)}\right)
$$

Where $d_{(i, s)} \geq 0$ is a continuous variable for the absolute deviation between the new calculated departure time and the initial departure time for train $i$ at event $s$ and $a_{(i, s)} \geq 0$ is also a continuous variable for the absolute deviation between the new calculated arrival time and the initial arrival time for train $i$ at event $s$. The corresponding deviation constraints are:

$$
\left|t_{(i, s)}^{B}-\hat{t}_{(i, s)}^{B}\right| \leq d_{(i, s)}
$$




$$
\left|t_{(i, s)}^{E}-\hat{t}_{(i, s)}^{E}\right| \leq a_{(i, s)}
$$

Constraints related to trains' arrival and departure times:

A new event $s+1$ for train $i$ starts right after finishing the previous event $s$.

$$
t_{(i, s)}^{E}=t_{(i, s+1)}^{B}
$$

Each event has a minimum duration separating its start and end times.

$$
t_{(i, s)}^{E} \geq t_{(i, s)}^{B}+t_{(i, s)}^{\min }
$$

Trains cannot depart from origin station earlier than the planned time. This constraint can be relaxed in the general problem of planning a timetable.

$$
t_{(i, 1)}^{B} \geq \hat{t}_{(i, 1)}^{B}
$$

If train $i$ is supposed to have a passenger stop at event $s$ then the new scheduled departure time should not be earlier than the planned departure time.

$$
t_{(i, s)}^{E} \geq \hat{t}_{(i, s)}^{E}
$$

Constraints for allowing trains to run safely in the opposite directions:

When trains are running in opposite directions in single and double tracks the following constraints should be applied.

When event $k$ happens before event $k+v$, as it is in the initial plan then:

$$
t_{\left(i_{(j, k+v)}, s_{(j, k+v)}\right)}^{B}-t_{\left(i_{(j, k)}, s_{(j, k)}\right)}^{E} \geq \gamma_{(j, k, v)} \cdot t_{j}^{c}-M \cdot\left(1-\gamma_{(j, k, v)}\right)
$$

When event $k$ happens after event $k+v$, the order is reversed compared to the initial plan.

$$
t_{\left(i_{(j, k)}, s_{(j, k)}\right)}^{B}-t_{\left(i_{(j, k+v)}, s_{(j, k+v)}\right)}^{E} \geq\left(1-\gamma_{(j, k, v)}\right) \cdot t_{j}^{c}-M \cdot \gamma_{(j, k, v)}
$$

When the order of trains is reversed compared to the initial order in double tracks. 


$$
t_{\left(i_{(j, k)}, s_{(j, k)}\right)}^{B}-t_{\left(i_{(j, k+v)}, s_{(j, k+v)}\right)}^{E} \geq \lambda_{(j, k, v)} \cdot t_{j}^{c}-M \cdot\left(1-\lambda_{(j, k, v)}\right)
$$

Constraints for allowing trains to run safely in the same direction:

When trains running in the same direction the following constraints should be applied.

For single and double tracks keeping the initial order:

$$
\begin{aligned}
& t_{\left(i_{(j, k+v)}, s_{(j, k+v)}\right)}^{B}-t_{\left(i_{(j, k)}, s_{(j, k)}\right)}^{B} \geq h_{\text {min }}-M \cdot\left(1-\gamma_{(j, k, v)}\right) \\
& t_{\left(i_{(j, k+v)}, s_{(j, k+v)}\right)}^{E}-t_{\left(i_{(j, k)}, s_{(j, k)}\right)}^{E} \geq h_{m i n}-M \cdot\left(1-\gamma_{(j, k, v)}\right)
\end{aligned}
$$

For double tracks with reverse order:

$$
\begin{aligned}
& t_{\left(i_{(j, k)}, s_{(j, k)}\right)}^{B}-t_{\left(i_{(j, k+v)}, s_{(j, k+v)}\right)}^{B} \geq h_{m i n}-M \cdot\left(1-\lambda_{(j, k, v)}\right) \\
& t_{\left(i_{(j, k)}, s_{(j, k)}\right)}^{E}-t_{\left(i_{(j, k+v)}, s_{(j, k+v)}\right)}^{E} \geq h_{m i n}-M \cdot\left(1-\lambda_{(j, k, v)}\right)
\end{aligned}
$$

\section{Constraints for track allocation:}

Each train $i$ should occupy only one track in each section $j$ along its journey.

$$
\sum_{q=1}^{C_{j(i, s)}} x_{\left(i, s, q_{(i, s)}\right)}=1 \quad C_{j_{(i, s)}} \geq 2
$$

In multi tracks, trains must not change tracks between two consecutive line sections unless there is a switch between the two tracks.

$$
x_{\left(i, s, q_{(i, s)}\right)}=x_{\left(i, s+1, q_{(i, s+1)}\right)} \quad C_{j_{(i, s)}} \geq 2
$$





\section{Chapter 3}

\section{Summary of the Included Papers}

Three papers are included in the thesis. In each paper a Basic Model is described and then adjusted for the indicated purposes. The basic model is based on the model described in section 2.3. However, there are some differences between the basic models used in paper I and II and paper III with respect to the corresponding assumptions.

Timetables that are generated by applying the basic model are called initial timetables and timetables generated by applying the adjusted (or modified) model are called modified timetables.

In the basic models for all papers, it is assumed that if a train has a scheduled stop at a station in the master timetable, then the allocated track has a sufficient platform length. The other assumption is that a train cannot depart earlier than the departure time for the first event.

\subsection{Paper I}

Improving Train Service Reliability by Applying an Effective Timetable Robustness Strategy

This paper presents a new headway-based strategy for improving robustness in railway timetables. The proposed strategy is implemented for real timetable cases and evaluated against small delays. A 
fundamental assumption for this work is that during the actual operations, the precision in operation of trains decreases with their travel time. The longer a train's journey is, the larger the risk that some small disturbances might occur during its journey. Hence, catching a fix-sized time slot in the timetable is easier the shorter the journey is. The assumption is not directly related to delays, but to the propagation of delays in the subsequent arrival times at the stations along a route.

In this paper we show how robustness can be improved in a given timetable by replacing a fix-sized time slot with a variable time slot, which increases with the travel time. We correlated the size of this new time slot to trains' travel times by introducing and implementing the parameter $\alpha$. This new time slot is calculated and reserved for each train and it is made sure that the headway between any pair of trains, which are running in the same direction and on the same track, is greater than this reserved time slot. The reserved time slot is also referred to as the scheduled minimum headway and the proposed idea is called Travel Time Dependent Scheduled Minimum Headway (TTDSMH).

Implementing large values for $\alpha$ leads to costs in terms of reductions in capacity utilization i.e. number of trains, changes in heterogeneity and reduction in average speed. We monitored the corresponding costs after implementing the proposed strategy and the results show that by increasing $\alpha$, the number of trains, heterogeneity and average speed are reduced. Moreover, by allowing a flexible ordering of trains, we can obtain larger headways between trains while having small reductions in capacity utilization, heterogeneity and speed. The modified timetables are also evaluated in different disturbance scenarios and the evaluation study shows that for disturbances up to 7 minutes, the modified timetables performed better (from robustness perspectives) in most of the experiments. The commercial solver Cplex is used to solve the problems.

The basic model in this paper contains some additional variables and constraints, compared to the basic model in section 2.3. The model is calibrated for technical minimum headway $h_{\text {min }}$ equal to 3 minutes. To implement TTDSMH strategy, headway is implemented as a variable and a set of constraints related to headways are developed and the existing headway constraints (constraints 2.13-2.16) are updated accordingly. The new constraints and variables can be found in paper I. 
Many of the previously proposed robustness strategies are too complicated to be realized and utilized by dispatchers. In real operations, dispatchers cannot make use of extra buffer times (or runtime margins) effectively if they cannot clearly observe them in the timetables. The proposed strategy in this paper is straight forward and can easily be understood and observed so that dispatchers can use it effectively in case of disturbances. However, in the experiments this method is applied for all types of trains but it can be separated by each train type. Furthermore, we assumed that the stop times at stations are necessary according to the master timetable. This assumption can become more accurate by studying the reasons of having stops at stations or by ignoring large stops from TTDMH calculations.

\subsection{Paper II}

\section{Robustness Improvements in a Train Timetable with Travel Time Dependent Minimum Headways}

This paper aims to verify the improvements in the robustness of some real timetables that are modified with respect to the idea of TTDSMH. The initial timetables are the annual master timetables that are calibrated for the purpose of the analysis and according to our assumptions. Both initial and modified timetables are evaluated for different disturbance scenarios from various performance indicators. Disturbance scenarios are as follows.

1) Single Delayed Train (SDT).

2) Speed Reduction for one single Train (SRT).

3) Speed Reduction for all the trains passing a specific Section (SRS).

Selected performance indicators include time deviation from the initial timetable, total delay, total number of delayed trains at destinations, number of trains that arrive at destinations with maximum 5 minutes delay and the number of overtaking violations compared to the initial timetable.

For experimental analysis of the model against delays, the basic model described in section 2.3 is applied by implementing some adjustments. The adjustments include the following restrictions: 1) the 
arrival and departure times of the delayed trains can only be postponed and earlier arrivals and departures are forbidden. All of the events before the occurrence of the delay, are fixed. 2) the order of trains can be fixed or flexible. The commercial solver Cplex is used to solve the problems.

In most of the experiments, timetables with TTDSMH outperform the initial timetables. In SDT scenarios, the modified timetables are more robust and this is more evident when the size of the delay is larger. In SRT scenario, the modified timetables have a better performance compared to the initial ones, however, the flexible trains' order also has a significant role to improve the performance. In SRS scenario, the modified timetables perform better but the improvements, compared to the initial timetables are small.

\subsection{Paper III}

\section{An Optimization Approach for On-Demand Railway Slot Al- location}

In this paper the problem of assessing and maintaining robustness when revising an existing timetable is studied. The results from papers I and II show that the applied optimization models need to be developed further to have faster computation time. This problem is also studied in this paper.

From the perspective of an infrastructure manager, we propose and experimentally evaluate an optimization-based approach for assessing and scheduling additional slot requests for traffic and urgent maintenance.

In paper III more operational planning details are included in the basic model. These details are:

- Forbidding simultaneous arrivals at stations where they are not supported. During the operations, simultaneous arrivals should not be allowed at stations without enough resources, i.e. stations on single track lines without extra tracks with sufficient length. To forbid simultaneous arrivals at stations and control the order of arrivals, a new binary variable $\omega_{(j, k, v)}$ is included, see also Törnquist Krasemann (2015). $\omega_{(j, k, v)}$ takes value one if event $k$ is happening at a station and occurs before event $k+v$ and zero otherwise. 
- Considering the lengths of trains and tracks at stations during track allocations so that when a train has a stop on a track at a station, the length of the allocated track should be sufficient. The objective function is also different and it contains components including the minimization of the number of track deviations and the travel time for the additional trains.

- Considering the value for technical minimum headway based on the data from Swedish Transport Administration (Trafikverket).

The adjusted model contains new parameters, variables and constraints for scheduling additional trains. These adjustments can be found in the appendix of paper III. The model is solved by using the commercial solver Gurobi.

The approach has been experimentally evaluated on a sub-network in Southern Sweden, where the traffic density is very high and it is challenging to assess and accommodate new slot requests close to the actual operation time.

When considering inserting additional trains, or maintenance, into an existing timetable, the resulting timetable will have different properties depending on the policy of insertion. That is, if there are multiple additional slots requests, the order in which they are inserted may affect the timetable as a whole but it may also affect the possibilities to accommodate the individual slot preferences. In this paper, we intend to investigate the effects of stepwise insertion, compared to simultaneous insertion, of a set of slot requests that partly compete for the same resources. Furthermore, in dense areas the insertion of additional trains may reduce the robustness of the timetable. One commonly discussed factor affecting the robustness is the time dependency between consecutive trains, i.e. the headway distance. Hence it is important to be aware of the consequences of each insertion policy on the properties of the revised timetable. A more in-depth discussion about robustness properties can be found in this paper.

The results show that inserting the additional trains simultaneously usually results in generating more optimal solutions. However, solving this type of problem is computationally challenging. We also observed that the existing robustness metrics cannot capture the essential properties of having more robust timetables. Therefore we propose measuring Channel Width, Channel Width Forward, Channel Width Behind and Track Switching when relevant. Channel Width $(\mathrm{CW})$ for a train can be defined as the time difference between the 
departure time of the train running in the front and the train running behind. It can be measured for each train at each location along its journey. We use Channel Width Behind (CWB) and Channel Width Front $(\mathrm{CWF})$ to measure the headway between the new inserted train and the trains running behind or in the front. $\mathrm{CW}$ is equal to the sum of CWB and CWF. CWB and CWF can be measured either at crucial locations, or at different locations in order to capture the minimum, maximum and average values along a train's journey. When trains are allowed to run on the opposite track the values for $\mathrm{CW}$ are not helpful enough and it is necessary to measure the frequency of track switching at line sections. Track Switching, specifies how often the trains swap side on the line sections. That is, how often trains are temporarily scheduled to operate on the opposite side of the line section, in order to e.g. overtake another train between two stations. A large value indicates a potential instability and increased risk for disturbances.

The experiments show that for certain types of scenarios, solving the corresponding timetabling problems is too time-consuming and there is a need to investigate and develop strategies for speeding up the solution process. Hence, we developed, implemented and evaluated boosting methods with focus on valid inequalities. Several valid inequalities are developed and their impact on the computation time is analyzed. Even though some of the proposed valid inequalities could reduce the size of the search space, they can also weaken the linear programming relaxation of the model and in some cases even increase the computation time. Further analysis shows that model is very sensitive to big $M$ values. 


\section{Chapter 4}

\section{Conclusions and Future Research}

\subsection{Conclusions}

In this thesis various timetabling problems are solved with respect to robustness by applying an existing MILP model which is further developed for each set of problems.

In paper I, in order to improve robustness in timetables, a headwaybased method is proposed based on train travel times. In this method, the headway between two trains is correlated to trains travel time. This method is evaluated and the corresponding results show that the modified timetables are more robust against small delays. Since assigning larger headways might lead to longer travel times and higher capacity consumptions, the impact of this method on capacity utilization, i.e. number of trains, heterogeneity and the speed of trains is evaluated and the results show that the corresponding effects are small. Furthermore, the proposed headway-based method is a straightforward method that can be realized easily in timetables by dispatchers when dealing with resolving delays.

In paper II, more evaluation analysis shows that even for large delays, i.e. when a section is under maintenance or when a train has a speed limit because of functional problems, the modified timetables still perform better than the initial ones. Furthermore, the flexibility of train orders has a significant impact on the results. The 
optimization-based method is fully deterministic and could be replaced by simulation methods to capture the stochasticity of the delays. However, although the delay analysis is not performed for stochastic delays, the worst case delay (according to our assumptions 7 minutes) is still tested. In the experiments this method is applied for all types of trains but it can be separated by each train type. Furthermore, we assumed that the stop times at stations are necessary according to the master timetable. This assumption can become more accurate by studying the reasons of having stops at stations or by ignoring large stops from TTDMH calculations.

In paper III, the problem of on-demand timetabling and the consequences of different planning policies are studied on robustness properties and capacity utilization. The analyses show that using the simultaneous insertion strategy results in generating timetables in which the travel times of the additional trains, is shorter, however, this type of problems is computationally demanding compared to the scenarios with stepwise insertion strategy. When inserting trains stepwise, the order of insertion matters and affects the capacity utilization and also the robustness properties of a timetable. We also observed that the existing robustness metrics cannot capture the essential properties of having more robust timetables. Therefore we propose measuring Channel Width, Channel Width Forward, Channel Width Behind and Track Switching.

Furthermore in paper III, the computation time is analyzed for a set of selected computationally hard problems. Several valid inequalities are proposed and their impact on the computation time is studied. Results show even though some of the proposed valid inequalities could reduce the size of the search space, they can also weaken the linear programming relaxation of the model and in some cases even increase the computation time. Additional analyses reveal that the computation time is very sensitive to the value of big $\mathrm{M}$.

\subsection{Future research}

Different robustness metrics have been introduced so far in the literature and the initial analyses show that they can be effective in practice. However, the generated robust timetables are only tested for simulated delays where it is assumed that the robustness properties are used effectively in order to limit the propagation of delays. In 
addition to the above, no before and after analysis has been conducted yet to test whether the implemented robustness properties are actually used by dispatchers during the occurrence of the disturbances. Hence, the real impact of the proposed robustness properties during real time operations is still unknown. The real impact of the proposed metrics on the robustness of timetables will be studied in future research.

We studied the consequences of two insertion strategies: simultaneous vs. stepwise insertion. However, during the revision process there are several possible decisions that need to be made. The presented research in this thesis showed how different insertion strategies might lead to different revised timetables. In the same manner, the consequences of all other possible decisions should also be studied. Furthermore, the criteria for selecting a revised timetable over the others is not well defined and different revised timetables might be selected with respect to various preferences. Hence, identifying the actual preferences of the operators and planners through surveys or interviews, and analyzing the consequences of various decisions are necessary.

The analysis of the computation time for different valid inequality scenarios showed that some of the valid inequalities can weaken the linear programming relaxation of the model which leads to an increase in the elapsed time for finding the first feasible solution. We did not provide any first feasible solution for the model when performing the experiments. The first feasible solution in different scenarios is found by the embedded heuristic methods in the applied commercial solver. This affects the total computation time and the first feasible solutions in different scenarios are not necessarily the same. To decrease the computation time, further boosting methods including providing a first feasible solution and developing solution algorithms can be studied in future research. 



\section{Bibliography}

Acuña-Agost, R. (2010). Mathematical modeling and methods for rescheduling trains under disrupted operations. PhD thesis, Université d'Avignon.

Andersson, E., Peterson, A., and Törnquist Krasemann, J. (2013). Quantifying railway timetable robustness in critical points. Journal of Rail Transport Planning 85 Management, 3(3):95 - 110.

Andersson, E. V. (2014). Assessment of robustness in railway traffic timetables. $\mathrm{PhD}$ thesis, Linköping University.

Andersson, E. V., Peterson, A., and Törnquist Krasemann, J. (2015). Reduced railway traffic delays using a MILP approach to increase Robustness in Critical Points. Journal of Rail Transport Planning $\&$ Management.

Aronsson, M. (2014). Erfarenheter från arbetet med sicsten, från forskning till prototyp. Slutrapport delprojekt Marackasen ST-1100, Trafikverket.

Aronsson, M., Bohlin, M., and Kreuger, P. (2009). MILP formulations of cumulative constraints for railway scheduling - A comparative study. volume 12 .

Assad, A. A. (1980). Models for rail transportation. Transportation Research Part A: General, 14(3):205-220.

Borndörfer, R., Grotschel, M., Lukac, S., and Mitusch, K. (2006). Auctioning Approach to Railway Slot Allocation, An. Competition and Regulation in Network Industries, 7:163.

Borndörfer, R. and Schlechte, T. (2007). Models for railway track allocation. volume 7 , pages $62-78$.

Borndörfer, R., Schlechte, T., and Weider, S. (2010). Railway track allocation by rapid branching. OpenAccess Series in Informatics: OASIcs, page 2746.

Brännlund, U., Lindberg, P. O., Nõu, A., and Nilsson, J.-E. (1998). Railway timetabling using Lagrangian relaxation. Transportation science, 32(4):358369.

Budai-Balke, G. (2009). Operations research models for scheduling railway infrastructure maintenance. Number 456. Rozenberg Publishers. 
Burdett, R. and Kozan, E. (2009). Techniques for inserting additional trains into existing timetables. Transportation Research Part B: Methodological, 43(8$9): 821-836$.

Cacchiani, V., Caprara, A., and Toth, P. (2010). Scheduling extra freight trains on railway networks. Transportation Research Part B: Methodological, 44(2):215231.

Cacchiani, V., Furini, F., and Kidd, M. (2016). Approaches to a real-world Train Timetabling Problem in a railway node. Omega (United Kingdom), 58:97-110.

Cacchiani, V., Huisman, D., Kidd, M., Kroon, L., Toth, P., Veelenturf, L., and Wagenaar, J. (2014). An overview of recovery models and algorithms for realtime railway rescheduling. Transportation Research Part B: Methodological, $63: 15-37$.

Cacchiani, V. and Toth, P. (2012). Nominal and robust train timetabling problems. European Journal of Operational Research, 219(3):727-737.

Caprara, A., Fischetti, M., and Toth, P. (2002). Modeling and Solving the Train Timetabling Problem. Operations Research, 50(5):851-861.

Caprara, A., Galli, L., Stiller, S., and Toth, P. (2014). Delay-Robust Event Scheduling. Operations Research, 62(2):274-283.

Caprara, A., Kroon, L., Monaci, M., Peeters, M., and Toth, P. (2007). Chapter 3 Passenger Railway Optimization. In Laporte, C. B. a. G., editor, Handbooks in Operations Research and Management Science, volume 14 of Transportation, pages 129-187. Elsevier.

Dewilde, T., Sels, P., Cattrysse, D., and Vansteenwegen, P. (2013). Robust railway station planning: An interaction between routing, timetabling and platforming. Journal of Rail Transport Planning \& Management, 3(3):68 - 77.

Edbring, T. (2014). Trångsektorsplan skåne, planeringsförutsättningar, tågplan t15. Technical report, The Swedish transport administration, Publication No.2014/17598.

Erol, B. (2009). Models for the train timetabling problem, master thesis. Master's thesis, University of Berlin, Department of Mathematics.

Eurostat (2014). Modal split of passenger transport (tsdtr210). Statistical Office of the European Union, http://epp.eurostat.ec.europa.eu/,2014-07-01.

Forsgren, M., Aronsson, M., and Gestrelius, S. (2013a). Maintaining tracks and traffic flow at the same time. Journal of Rail Transport Planning $\mathscr{B}$ Management, 3(3):111-123.

Forsgren, M., Aronsson, M., and Gestrelius, S. (2013b). Towards shorter lead times in railway timetabling in sweden. In Proceedings of IEEE Conference on Intelligent Transportation Systems, ITSC, pages 1053-1058. 
Forsgren, M., Aronsson, M., Gestrelius, S., and Dahlberg, H. (2012). Using timetabling optimization prototype tools in new ways to support decision making. Computers in Railways XIII: Computer System Design and Operation in the Railway and Other Transit Systems, 127:439-450.

Gestrelius, S., Aronsson, M., Forsgren, M., and Dahlberg, H. (2012). On the delivery robustness of train timetables with respect to production replanning possibilities. In Proceedings of the 2nd International Conference on Road and Rail Infrastructure (CETRA), Dubrovnik, Croatia, pages 823-830.

Harrod, S. (2013). Auction pricing of network access for North American railways. Transportation Research Part E: Logistics and Transportation Review, 49(1):176-189.

Harrod, S. and Schlechte, T. (2013). A direct comparison of physical block occupancy versus timed block occupancy in train timetabling formulations. Transportation Research Part E: Logistics and Transportation Review, 54:50-66.

Harrod, S. S. (2012). A tutorial on fundamental model structures for railway timetable optimization. Surveys in Operations Research and Management Science, 17(2):85-96.

Heydar, M., Petering, M. E. H., and Bergmann, D. R. (2013). Mixed integer programming for minimizing the period of a cyclic railway timetable for a single track with two train types. Computers $\mathcal{E}$ Industrial Engineering, 66(1):171-185.

Jovanović, P., Kecman, P., Bojović, N., and Mandić, D. (2016). Optimal allocation of buffer times to increase train schedule robustness. European Journal of Operational Research, pages -.

Khoshniyat, F. and Peterson, A. (2015). Robustness improvements in a train timetable with travel time dependent minimum headways. In Proceedings of 6th International Conference on Railway Operations Modelling and Analysis RailTokyo.

Klabes, S. G. (2010). Algorithmic railway capacity allocation in a competitive European railway market. $\mathrm{PhD}$ thesis, PhD thesis, RWTH Aachen, 2010. Cited on pages xxv, 11, 13, 29, 31, 39, 40, 57, 61, 62, 90 .

Konrad, P. (2014). Riktlinjer täthet mellan tåg. Technical report, The Swedish transport administration, Publication No.TRV 2014/97400.

Kraay, D. R. and Harker, P. T. (1995). Real-time scheduling of freight railroads. Transportation Research Part B: Methodological, 29(3):213-229.

Kroon, L., Huisman, D., and Maróti, G. (2008a). Optimization models for railway timetabling. Hansen and Pachl (Eds.): Railway Timetable $\&$ Traffic. Analysis, Modelling, Simulation, Hamburg: Eurailpress, pages 135-154.

Kroon, L., Huisman, D., and Maróti, G. (2008b). Optimisation models for railway timetabling. In Hansen, A. and Pachl, J., editors, Railway timetable and traffic, analysis, modelling, simulation. Eurailpress, Hamburg. 
Kroon, L., Maróti, G., Helmrich, M., Vromans, M., and Dekker, R. (2008c). Stochastic improvement of cyclic railway timetables. Transportation Research Part B: Methodological, 42(6):553 - 570.

Kroon, L. G., Dekker, R., and Vromans, M. J. C. M. (2007). Cyclic Railway Timetabling: A Stochastic Optimization Approach. In Geraets, F., Kroon, L., Schoebel, A., Wagner, D., and Zaroliagis, C. D., editors, Algorithmic Methods for Railway Optimization, number 4359 in Lecture Notes in Computer Science, pages 41-66. Springer Berlin Heidelberg. DOI: 10.1007/978-3-540-74247-0_2.

Kroon, L. G., Edwin Romeijn, H., and Zwaneveld, P. J. (1997). Routing trains through railway stations: complexity issues. European Journal of Operational Research, 98(3):485-498.

Lamorgese, L. and Mannino, C. (2013). The track formulation for the train dispatching problem. Electronic Notes in Discrete Mathematics, 41:559-566.

Lamorgese, L. and Mannino, C. (2015). An Exact Decomposition Approach for the Real-Time Train Dispatching Problem. Operations Research, 63(1):48-64.

Lidén, T. (2016). Towards concurrent planning of railway maintenance and train services, volume 1746. Linköping University Electronic Press.

Liebchen, C., Schachtebeck, M., Schöbel, A., Stiller, S., and Prigge, A. (2010). Computing delay resistant railway timetables. Computers \&S Operations Research, 37(5):857-868.

Louwerse, I. and Huisman, D. (2014). Adjusting a railway timetable in case of partial or complete blockades. European Journal of Operational Research, $235(3): 583-593$.

Lusby, R. M., Larsen, J., Ehrgott, M., and Ryan, D. (2009). Railway track allocation: models and methods. OR Spectrum, 33(4):843-883.

Mannino, C. (2011). Real-time traffic control in railway systems. In 11th Workshop on Algorithmic Approaches for Transportation Modelling, Optimization, and Systems, volume 20 of OpenAccess Series in Informatics (OASIcs), pages 1-14. Schloss Dagstuhl-Leibniz-Zentrum fuer Informatik.

Meng, L. and Zhou, X. (2014). Simultaneous train rerouting and rescheduling on an N-track network: A model reformulation with network-based cumulative flow variables. Transportation Research Part B: Methodological, 67:208-234.

Pellegrini, P., Marliere, G., Pesenti, R., and Rodriguez, J. (2015). RECIFE-MILP: An Effective MILP-Based Heuristic for the Real-Time Railway Traffic Management Problem. IEEE Transactions on Intelligent Transportation Systems, 16(5):2609-2619.

Pellegrini, P., Marlière, G., and Rodriguez, J. (2014). Optimal train routing and scheduling for managing traffic perturbations in complex junctions. Transportation Research Part B: Methodological, 59:58-80. 
Perennes, P. (2014). Use of combinatorial auctions in the railway industry: Can the "invisible hand" draw the railway timetable? Transportation Research Part A: Policy and Practice, 67:175-187.

Robenek, T., Jiang Hang, C., and Bierlaire, M. (2014). The ideal train timetabling problem. In 14th Swiss Transportation Research Conference, number EPFLCONF-199480.

Salido, M., Barber, F., and Ingolotti, L. (2008). Robustness in railway transportation scheduling. In Proceedings of the World Congress on Intelligent Control and Automation (WCICA), pages 2833-2837.

Schöbel, A. (2001). A Model for the Delay Management Problem based on Mixed-Integer-Programming. Electronic Notes in Theoretical Computer Science, 50(1):1-10.

Törnquist, J. and Persson, J. A. (2007). N-tracked railway traffic re-scheduling during disturbances. Transportation Research Part B: Methodological, 41(3):342362.

Törnquist Krasemann, J. (2012). Design of an effective algorithm for fast response to the re-scheduling of railway traffic during disturbances. Transportation Research Part C: Emerging Technologies, 20(1):62-78.

Törnquist Krasemann, J. (2015). Computational decision-support for railway traffic management and associated configuration challenges: An experimental study. Journal of Rail Transport Planning and Management, 5(3):95-109.

Vromans, M. (2005). Reliability of Railway Systems. PhD thesis, Erasmus University Rotterdam.

Vromans, M., Dekker, R., and Kroon, L. (2006). Reliability and heterogeneity of railway services. European Journal of Operational Research, 172(2):647-665.

Zwaneveld, P. J., Kroon, L. G., Romeijn, H. E., Salomon, M., Dauzere-Peres, S., Van Hoesel, S. P., and Ambergen, H. W. (1996). Routing trains through railway stations: Model formulation and algorithms. Transportation science, $30(3): 181-194$. 



\section{Papers}

The articles associated with this thesis have been removed for copyright reasons. For more details about these see:

http://urn.kb.se/resolve?urn=urn:nbn:se:liu:diva-132920 